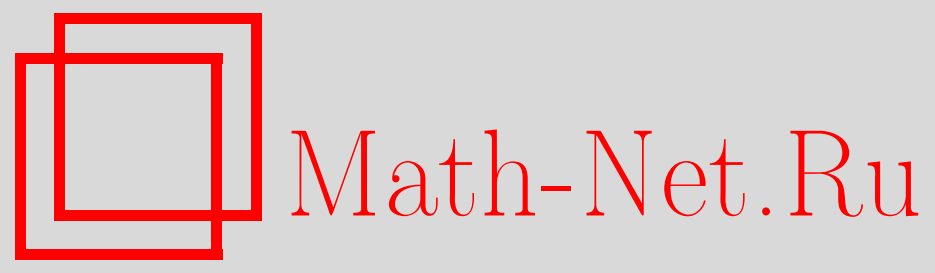

Д. Н. Семёновых, Обобщение квадратично-вычетных кодов на случай вычетов третьей и четвертой степени, Дискрет. матем., 2005, том 17, выпуск 4, 143-149

DOI: https://doi.org/10.4213/dm136

Использование Общероссийского математического портала Math-Net.Ru подразумевает, что вы прочитали и согласны с пользовательским соглашением http://www . mathnet.ru/rus/agreement

Параметры загрузки:

IP : 18.234 .156 .22

26 апреля 2023 г., 13:00:23

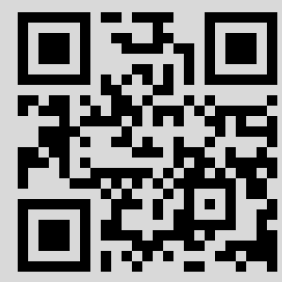




\title{
Обобщение квадратично-вычетных кодов на случай вычетов третьей и четвертой степени
}

\author{
() 2005 г. Д. Н. Семёновых
}

\begin{abstract}
В статье рассмотрено обобщение квадратично-вычетных кодов на случаи вычетов более высоких степеней. В результате такого обобщения улучшается относительная скорость передачи при использовании таких кодов в сравнении с квадратичновычетными кодами, правда, при некотором ухудшении нижней оценки на кодовое расстояние. Рассмотрено построение порождающих многочленов таких кодов, что позволяет явно выписывать их порождающую матрицу.
\end{abstract}

Пусть $p$ - нечетное простое число, $n \in \mathrm{N}, n \geqslant 2$.

Определение 1. Элемент $a \in G F(p)^{*}$ называется вычетом степени $n$ по модулю $p$, если сравнение

$$
x^{n} \equiv a \quad(\bmod p)
$$

разрешимо в $G F(p)^{*}$.

Вычеты степени $n$ по модулю $p$ будем для кратности называть также $n$-вычетами по модулю $p$.

Пусть $g$ - произвольный порождаюший элемент группы $G F(p)^{*}$, так что $G F(p)^{*}=$ $\langle g\rangle_{p-1}$. Тогда для элемента $x \in G F(p)^{*}$ справедливо сравнение $x \equiv g^{y}(\bmod p)$ для некоторого $y \in\{0,1, \ldots, p-2\}$. Таким образом, если $a \equiv g^{m}(\bmod p)$, то сравнение (1) принимает вид $g^{n y} \equiv g^{m}(\bmod p)$, то есть $n y \equiv m(\bmod p-1)$. Последнее сравнение, как известно, разрешимо, если наибольший общий делитель $h=(n, p-1)$ делит $m$. В этом случае оно имеет ровно $h$ различных решений.

Будем предполагать, что $p$ и $n$ выбраны таким образом, что $h \geqslant 2$. Рассмотрим следующие $h$ классов:

$$
\begin{aligned}
& Q_{i}=\left\{r \in G F(p)^{*} \mid r=g^{k}, k \in \mathbf{Z}, 0 \leqslant k \leqslant p-2, k \equiv i \quad(\bmod h)\right\}, \\
& i=0,1, \ldots, h-1 \text {. }
\end{aligned}
$$

Тогда класс $Q_{0}$ состоит из всех $n$-вычетов по модулю $p$.

Предположим теперь дополнительно, что число $p$ выбрано таким образом, что $2 \in Q_{0}$, то есть 2 является вычетом степени $n$ по модулю $p$. В случаях $h=3$ или $h=4$ (а в дальейшем мы более подробно остановимся именно на кубических и биквадратичных вычетах) известно (см., например, [2]), что это требование равносильно представимости 
числа $p$ в виде $p=A^{2}+27 B^{2}, A, B \in \mathbf{Z}$ при $h=3$ и $p=A^{2}+64 B^{2}, A, B \in \mathbf{Z}$ при $h=4$.

Пусть $R_{p}=G F(2)[x] /\left(x^{p}-1\right)$, тогда произвольный идеал в данном фактор-кольце представляет собой циклический код длины $p$. Найдем конечное поле характеристики 2 , в котором многочлен $x^{p}-1$ раскладывается на линейные множители. Так как $(2, p)=1$, существует такое число $t \in \mathrm{N}$, что выполнено сравнение $2^{t} \equiv 1(\bmod p)$, то есть $p$ делит $2^{t}-1$, следовательно, многочлен $x^{p}-1$ делит многочлен $x^{2^{t}-1}-1$. Многочлен $x^{2^{t}-1}-1$ имеет своими однократными корнями все ненулевые элементы поля $G F\left(2^{t}\right)$. Поэтому многочлен $x^{p}-1$ раскладывается в поле $G F\left(2^{t}\right)$ на линейные множители. Его корни образуют циклическую группу простого порядка корней степени $p$ из единищы в $G F\left(2^{t}\right)^{*}$. Выберем в этой группе некоторый примитивный корень $\alpha$.

Для каждого числа $i \in\{0,1, \ldots, h-1\}$ рассмотрим многочлен

$$
q_{i}(x)=\prod_{r \in Q_{i}}\left(x-\alpha^{r}\right) .
$$

Предложение 1. Для многочлена $q_{i}(x)$ справедливы соотношения

$$
\begin{aligned}
\operatorname{deg} q_{i}(x) & =\frac{p-1}{h}, & & i=0,1, \ldots, h-1, \\
q_{i}(x) & \in G F(2)[x], & i & =0,1, \ldots, h-1 .
\end{aligned}
$$

Доказательство. Первое утверждение следует из того, что класс $Q_{i}$ содержит $(p-1) / h$ элементов.

Докажем второе утверждение. Если $r \in Q_{i}$, то $r \equiv g^{k}(\bmod p)$, где $k \equiv i(\bmod h)$ (здесь и всюду далее рассматриваются наименьшие неотрицательные вычеты по модулю $h)$. Так как $2 \in Q_{0}$, то $2 \equiv g^{k_{0}}(\bmod p)$, где $k_{0} \equiv 0(\bmod h)$. Поэтому $2 r \equiv q^{k+k_{0}}$ $(\bmod p)$, где $k+k_{0} \equiv i(\bmod h)$, то есть $2 r \in Q_{i}$. При этом умножение на 2 осуществляет взаимно однозначное отображение $Q_{i}$ в себя.

Это означает, что корни многочлена $q_{i}(x)$ образуют множество всех элементов, сопряженных элементу $\alpha^{r}$ над полем $G F(2)$, причем все они являются корнями кратности 1 , откуда и следует доказываемое утверждение.

Очевидно, что $q_{i}(x)$ делит $x^{p}-1$, и из предложения 1 следует, что можно рассматривать коды $\mathfrak{L}_{i}=\left\langle q_{i}(x)\right\rangle, i=0,1, \ldots, h-1$, являющиеся главными идеалами в кольце $R_{p}$. Более того , можно утверждать (см. [1]), что многочлен $q_{i}(x)$ является многочленом наименњшей степени, входящим в идеал $\mathfrak{L}_{i}$.

Предложкение 2 (Свойства кодов $\left.\mathfrak{L}_{i}\right)$. Для кодов $\mathfrak{L}_{i}$ справедливы следующие утверждения.

(1) Длина кода (длина каждого кодового слова) равна р.

(2) Размерность кода равна $p-\operatorname{deg} q_{i}(x)=p-(p-1) / h$, а относительная скорость передачи равна $1-1 / h+1 /(p h)$.

(3) Все коды $\mathfrak{R}_{i}$ друг другу эквивалентны и получаются друг из друга некоторой перестановкой координатных позичий.

(4) Кодовое расстояние $d$ удовлетворяет неравенству $d \geqslant p^{1 / h}$. 
Доказательство. Пункты (1) и (2) очевидны.

Докажем (3). Покажем, что каждый код $\mathfrak{L}_{i}$ при $i=1, \ldots, h-1$ получается из кода $\mathfrak{L}_{0}$ некоторой перестановкой координатных позищий. Выберем произвольное $s \in Q_{h-i}$. Пусть $a(x) \in \mathfrak{L}_{0}$. Покажем, что $a\left(x^{s}\right) \in \mathfrak{L}_{i}$. Действительно, если $a(x) \in \mathfrak{L}_{0}$, то $q_{0}(x)$ делит $a(x)$ и поэтому $a\left(\alpha^{r}\right)=0$ для любого $r \in Q_{0}$.

Для любого $t \in Q_{i}$ справедливо равенство $a\left(\alpha^{s t}\right)=0$, так как st $\in Q_{0}$. Поэтому $a\left(x^{s}\right) \in \mathfrak{R}_{i}$.

Поскольку данное отображение, очевидно, инъективно и является отображением линейных пространств одинаковой размерности, оно и сюръективно, и следовательно, утверждение доказано.

Докажем (4). Пусть $d$ - кодовое расстояние кода $\mathfrak{L}_{0}$, выберем кодовое слово $a(x) \in \mathfrak{L}_{0}$ наименьшего веса $d$. Для произвольного $s_{i} \in Q_{h-i}$ рассмотрим многочлен $a\left(x^{s_{i}}\right), i=1, \ldots, h-1$. Тогда, согласно пункту (3), многочлен $a\left(x^{s_{i}}\right) \in \mathfrak{L}_{i}$ и является в этом коде словом веса $d$, так как получен из $a(x)$ некоторой перестановкой координатных позищий.

Рассмотрим многочлен

$$
a(x) a\left(x^{s_{1}}\right) a\left(x^{s_{2}}\right) \ldots a\left(x^{s_{h-1}}\right) \equiv r(x) \quad\left(\bmod x^{p}-1\right), \quad \operatorname{deg}(r(x)) \leqslant p-1 .
$$

Ясно, что $\prod_{r \in Q_{0}}\left(x-\alpha^{r}\right) \prod_{r \in Q_{1}}\left(x-\alpha^{r}\right) \ldots \prod_{r \in Q_{h-1}}\left(x-\alpha^{r}\right)$ делит $r(x)$. Данное произведение равно

$$
\frac{x^{p}-1}{x-1}=\sum_{i=0}^{p-1} x^{i}
$$

и $\operatorname{deg}(r(x)) \leqslant p-1$, поэтому

$$
r(x)=\sum_{i=0}^{p-1} x^{i},
$$

то есть $r(x)$ - слово веса Хэмминга $w \mathrm{t}(r(x))=p$.

С другой стороны, $\operatorname{wt}(r(x)) \leqslant d^{h}$, откуда получаем неравенство $d^{h} \geqslant p$, равносильное доказываемому неравенству.

Предложение 2 доказано.

Наряду с кодами $\mathfrak{L}_{i}$ можно рассматривать расширенные коды $\overline{\mathfrak{L}}_{i}=\left\langle(x-1) q_{i}(x)\right\rangle$. Очевидно, что $\overline{\mathfrak{l}}_{i} \subset \mathfrak{L}_{i}$ при всех $i=0,1, \ldots, h-1$. Более того, легко видеть, что слова кода $\overline{\mathfrak{L}}_{i}=\left\langle(x-1) q_{i}(x)\right\rangle$ - это в точности все слова четного веса кода $\mathfrak{L}_{i}$, поскольку соответствующие многочлены обращаются в ноль в точке $x=1$.

Знание явного вида порождающего многочлена кода позволяет выписывать порождающую матрицу этого кода (см. [1]). В силу того, что явное вычисление коэффициентов порождающего многочлена довольно сложно, будем искать некоторый другой многочлен, лежащий в данном коде (не обязательно минимальной степени), порождающий данный код. Обычно в теории кодирования в качестве такого многочлена рассматривают порождаюший идемпотент кода.

Определение 2. Многочлен $E(x) \in R_{p}$ - идемпотент, если в $R_{p}$ выполнено равенство $E^{2}(x)=E(x)$.

Далее будем использовать приведенные ниже известные результаты (доказательства см. в [1]). 
Предложение 3. Для любого ииклического кода существует единственный идемпотент, порождаючий этот код.

Предложение 4. Пусть $\mathfrak{L}=\langle q(x)\rangle$ - циклический код. Идемпотент $E(x)$ порождаeт код $\mathfrak{L}$ тогда и только тогда, когда для любого примитивного корня $\alpha$ степени $p$ из единичы значения $E\left(\alpha^{r}\right)$ и $q\left(\alpha^{r}\right)$ равны или не равны нулю одновременно для всех $r \in\{0,1, \ldots, h-1\}$.

Предложение 5. Многочлен $E(x)$ - идемпотент тогда и только тогда, когда для любого примитивного корня $\alpha$ степени р из единицы значение $E(\alpha) \in\{0,1\}$.

Рассмотрим многочлены

$$
T_{i}(x)=\sum_{r \in Q_{i}} x^{r}, \quad i=0,1, \ldots, h-1 .
$$

В следующем предложении в качестве индексов многочленов $T_{i}$ будут рассматриваться наименышие неотрицательные вычеты по модулю $h$.

Предложение 6. Многочлены $T_{i}, i=0,1, \ldots, h-1$, обладают следующими свойствами.

(1) $T_{i}^{2}(x)=T_{i}(x)$ в $R_{p}$.

(2) Для любого примитивного корня $\alpha$ степени р из единицы

$$
\sum_{i=0}^{h-1} T_{i}(\alpha)=1
$$

(3) Для любого $i=0,1, \ldots, h-1$ справедливо включение $T_{i}(\alpha) \in\{0,1\}$.

(4) Пусть $r \in Q_{j}, j=0,1, \ldots, h-1$. Тогда при всех $i=0,1, \ldots, h-1$ справедливо равенство $T_{i}\left(\alpha^{r}\right)=T_{i+j}(\alpha)$, то есть значение $T_{i}\left(\alpha^{r}\right)$ при фиксированном $i$ зависит только от класса $Q_{j}$, которому принадлежит показатель $r$.

Доказательство. Для доказательства первого утверждения возведем многочлен в квадрат и, пользуясь тем, что умножение на 2 осуществляет взаимно однозначное отображение $Q_{i}$ в себя (см. доказательство предложения 1 ), получаем, что в $R_{p}$

$$
T_{i}^{2}(x)=\sum_{r \in Q_{i}} x^{2 r}=\sum_{s \in Q_{i}} x^{s}=T_{i}(x) .
$$

Для доказательства (2) заметим, что

$$
\sum_{i=0}^{h-1} T_{i}(\alpha)=\sum_{k=1}^{p-1} \alpha^{k}=\alpha^{0}=1
$$

Утверждение (3) следует из пункта (1) с использованием предложения 5.

Утверждение (4) следует из справедливости равенств

$$
T_{i}\left(\alpha^{r}\right)=\sum_{s \in Q_{i}}\left(\alpha^{r}\right)^{s}=\sum_{t \in Q_{i+j}} \alpha^{t}=T_{i+j}(\alpha)
$$

Предложение 6 доказано. 
Определение 3. Скажем, что семейство кодов $\mathfrak{l}_{i}$ имеет распределение $\left(a_{0}, a_{1}, \ldots, a_{h-1}\right)$, $a_{i} \in\{0,1\}$, если для некоторого примитивного корня $\alpha$ из единицы степени $p$ справедливы равенства

$$
T_{0}(\alpha)=a_{0}, T_{1}(\alpha)=a_{1}, \ldots, T_{h-1}(\alpha)=a_{h-1} .
$$

Набор $\left(a_{0}, a_{1}, \ldots, a_{h-1}\right)$ определен с точностью до циклического сдвига его элементов. Действительно, взяв в качестве примитивного корня степени $p$ из единищы элемент $\alpha^{r}$, где $r \in Q_{i}$, получим, согласно утверждению (4) предложения 6, распределение, в котором элементы получаются циклическим сдвигом первоначального распределения на $i$ позиций вправо. Поэтому распределения можно рассматривать с точностью до описанного циклического сдвига, выбирая нужным образом примитивный корень $\alpha$.

Заметим также, что, согласно утверждению (2) предложения 6, любое распределение семейства кодов $\mathfrak{L}_{i}$ может содержать лишь нечетное число единиц. Более того, оно не может состоять из всех единиц. Действительно, в противном случае получим, что $T_{0}\left(\alpha^{r}\right)=1$ для всех $r, 0<r \leqslant p-1$, откуда следует, что $x^{p-1}+\ldots+x+1$ делит $T_{0}(x)-1$ в $R_{p}$ или $\left(T_{0}(x)-1\right)(x-1) \equiv 0\left(\bmod x^{p}-1\right)$, что невозможно, так как $T_{0}(x)$ имеет $(p-1) / h$ слагаемых, $h \geqslant 2$ и $\operatorname{deg} T_{0}(x) \leqslant p-1$.

В случае квадратичных, кубических и биквадратичных вычетов (то есть при $h=2$, $h=3$ и $h=4$ соответственно), зная параметры кода, можно указать соответствующее распределение и выписать порождающие идемпотенты.

Теорема 1 (случай квадратичных вычетов). При $h=2$ примитивный корень $\alpha$ можно выбрать таким образом, что порождающими идемпотентами $E_{i}(x)$ кодов $\mathfrak{L}_{i}$ и порождаючими идемпотентами $\bar{E}_{i}$ кодов $\overline{\mathfrak{L}}_{i}$ будут являться, соответственно, многочлены

$$
\begin{array}{ll}
E_{0}(x)=T_{1}(x)+1, & E_{1}(x)=T_{0}(x)+1, \\
\bar{E}_{0}(x)=T_{0}(x), & \bar{E}_{1}(x)=T_{1}(x)
\end{array}
$$

при $(p-1) / 2$ четном $u$

$$
\begin{array}{ll}
E_{0}(x)=T_{0}(x), & E_{1}(x)=T_{1}(x), \\
\bar{E}_{0}(x)=T_{1}(x)+1, & \bar{E}_{1}(x)=T_{0}(x)+1
\end{array}
$$

при $(p-1) / 2$ нечетном.

Доказательство см. в [1].

Теорема 2 (случай кубических вычетов). При $h=3$ примитивный корень $\alpha$ можно выбрать таким образом, что порождающими идемпотентами $E_{i}(x)$ кодов $\mathfrak{R}_{i}$ и порождающими идемпотентами $\bar{E}_{i}(x)$ кодов $\overline{\mathfrak{L}}_{i}$ будут являться, соответственно, многочлены

$$
E_{i}(x)=T_{3-i}(x)+1, \quad \bar{E}_{i}(x)=\sum_{j \neq 3-i} T_{j}(x) .
$$

Доказательство. Из предыдущих рассуждений о свойствах распределений кодов следует, что код имеет распределение $(1,0,0)$. Проверим, что указанные идемпотенты действительно порождают код.

Пусть $r \in Q_{l}$. Тогда выполнены равенства

$$
\begin{aligned}
& E_{i}\left(\alpha^{r}\right)=T_{3-i}\left(\alpha^{r}\right)+1=T_{3-i+l}(\alpha)+1=\delta_{i}^{l}+1, \\
& \bar{E}_{i}\left(\alpha^{r}\right)=\sum_{j \neq 3-i} T_{j}\left(\alpha^{r}\right)=\sum_{j \neq 3-i} T_{j+l}(\alpha)=\sum_{k \neq 3-i+l} T_{k}(\alpha)=\delta_{i}^{l}+1,
\end{aligned}
$$


где $\delta_{i}^{l}$ - символ Кронекера. По построению 3 делит $p-1$, поэтому $(p-1) / 3$ - четное число и $E_{i}(1)=1, \bar{E}_{i}(1)=0$.

Теорема доказана.

Теорема 3 (случай биквадратичных вычетов). При $h=4$ примитивный корень $\alpha$ можно выбрать таким образом, что

(1) в случае $p \equiv 1(\bmod 16)$ код $\mathfrak{L}_{0}$ имеет распределение $(1,0,0,0)$ и идемпотенты

$$
E_{i}(x)=T_{4-i}(x)+1, \quad \bar{E}_{i}(x)=\sum_{j \neq 4-i} T_{j}(x),
$$

(2) в случае $p \equiv 9(\bmod 16)$ код $\mathfrak{L}_{0}$ имеет распределение $(0,1,1,1)$ и идемпотенты

$$
E_{i}(x)=\sum_{j \neq 4-i} T_{j}(x)+1, \quad \bar{E}_{i}(x)=T_{4-i}(x) .
$$

Доказательство. Заметим, что в теореме разобраны все возможные случаи для простых чисел $p$ таких, что 4 делит $p-1$ и $p=A^{2}+64 B^{2}, A, B \in Z$, то есть 2 является вычетом степени 4 по модулю $p$.

Пусть $p \equiv 1(\bmod 16)$. Предположим, что соответствуюший код имеет распределение $(0,1,1,1)$. Рассмотрим сумму

$$
S(x)=\sum_{0 \leqslant i<j \leqslant 3} T_{i}(x) T_{j}(x)
$$

тогда $S(\alpha)=1$ в $G F(2)$. С другой стороны,

$$
S(\alpha)=\sum_{r \in Q_{k}, s \in Q_{l}, k<l} \alpha^{r+s} .
$$

Здесь индексы $(r, s)$ образуют всевозможные неупорядоченные пары элементов из $G F(p)^{*}$, лежащих в разных классах, при этом каждая пара элементов берется по одному разу. Сумма $r+s$ рассматривается по модулю $p$.

Если для произвольного элемента $y \in G F(p)^{*}$ справедливо представление $y \equiv r+s$ $(\bmod p)$, где $r$ и $s$ из разных классов, то для произвольного элемента $x \in G F(p)^{*}, x \neq y$, справедливо сравнение $x \equiv g^{k} y(\bmod p)$ при некотором $k, 0<k \leqslant p-2$. Тогда

$$
x \equiv g^{k}(r+s) \equiv \bar{r}+\bar{s} \quad(\bmod p),
$$

где $\bar{r}$ и $\bar{s}$ тоже будут лежать в разных классах. Таким образом, отображение $y \mapsto x=g^{k} y$ осуществляет взаимно однозначное соответствие между представлениями элементов из $G F(p)^{*}$ в виде суммы двух элементов $r$ и $s$ из разных классов. Значит, для каждого элемента $y \in G F(p)^{*}$ число таких представлений одинаково, обозначим его через $L$.

Поскольку $p \equiv 1(\bmod 16)$, справедливо сравнение $(-1)^{(p-1) / 4} \equiv 1(\bmod p)$. Следовательно, $-1 \in Q_{0}$. Поэтому элементы $i$ и $-i(\bmod p)$ лежат в одном классе, то есть в сумме (2) показатели степеней никогда не равны нулю по модулю $p$.

Тогда

$$
S(\alpha)=\sum_{r \in Q_{k}, s \in Q_{l}, k<l} \alpha^{r+s}=L \sum_{i=1}^{p-1} \alpha^{i}=L .
$$


Так как $S(\alpha)=1$ в $G F(2)$, число $L$ нечетно.

Подсчитаем теперь число слагаемых, входящих в сумму (2). Их число, с одной стороны, равно $L(p-1)$, а с другой стороны, $C_{4}^{2}((p-1) / 4)((p-1) / 4)$.

Получаем равенство в натуральных числах $L(p-1)=6((p-1) / 4)^{2}$. Вводя обозначение $(p-1) / 4=4 k$, приходим к равенству $L=6 k$, в котором $L-$ нечетное число. Полученное противоречие означает, что в случае $p \equiv 1(\bmod 16)$ код имеет распределение $(1,0,0,0)$.

Пусть теперь $p \equiv 9(\bmod 16)$ и предположим, что код имеет распределение $(1,0,0,0)$. Рассматривая опять сумму (2), получим, что $S(\alpha)=0$ в $G F(2)$. В данном случае снова $(-1)^{(p-1) / 4} \equiv 1(\bmod p)$. Следовательно, $-1 \in Q_{0}$. Проведя аналогичные рассуждения, получим равенство в натуральных числах $L(p-1)=6((p-1) / 4)^{2}$, которое после замены $(p-1) / 4=2 k$, где $k$ - нечетное число, приводится к виду $L=3 k$, в котором $L$ - четное число. Полученное противоречие означает означает, что в данном случае код имеет распределение $(0,1,1,1)$.

Проверим теперь, что указанные в условии теоремы многочлены действительно являются порождающими идемпотентами соответствующих кодов. Действительно, при $p \equiv 1$ $(\bmod 16)$ для $r \in Q_{l}$ справедливы соотношения

$$
\begin{aligned}
& E_{i}\left(\alpha^{r}\right)=T_{4-i}\left(\alpha^{r}\right)+1=T_{4-i+l}(\alpha)+1=\delta_{i}^{l}+1, \\
& \bar{E}_{i}\left(\alpha^{r}\right)=\sum_{j \neq 4-i} T_{j}\left(\alpha^{r}\right)=\sum_{j \neq 4-i} T_{j+l}(\alpha)=\sum_{j \neq 4-i+l} T_{j}(\alpha)=\delta_{i}^{l}+1,
\end{aligned}
$$

где $\delta_{i}^{l}$ - символ Кронекера. Далее, в данном случае $(p-1) / 4$ - четное число, поэтому

$$
E_{i}(1)=T_{4-i}(1)+1=1, \quad \bar{E}_{i}(1)=\sum_{j \neq 4-i} T_{j}(1)=0 .
$$

Применение предложения 4 доказывает теорему для данного случая.

Если теперь $p \equiv 9(\bmod 16)$, то аналогично для $r \in Q_{l}$ получаем соотношения

$$
\begin{aligned}
& E_{i}\left(\alpha^{r}\right)=\sum_{j \neq 4-i} T_{j}\left(\alpha^{r}\right)+1=\sum_{j \neq 4-i} T_{j+l}(\alpha)+1=\sum_{j \neq 4-i+l} T_{j}(\alpha)+1=\delta_{i}^{l}+1, \\
& \bar{E}_{i}\left(\alpha^{r}\right)=T_{4-i}\left(\alpha^{r}\right)=T_{4-i+l}(\alpha)=\delta_{i}^{l}+1 .
\end{aligned}
$$

Наконец, так как в данном случае также $(p-1) / 4$ - четное число, справедливы равенства

$$
E_{i}(1)=\sum_{j \neq 4-i} T_{j}(1)+1=1, \quad \bar{E}_{i}(1)=T_{4-i}(1)=0 .
$$

Согласно предложенню 4, рассматриваемые многочлены действительно являются порожденными идемпотентами. Теорема 3 полностью доказана.

\section{Список литературы}

1. Мак-Вильямс Ф. Дж., Слоэн Н. Д. А., Коды, корректирующие ошибки. Связь, Москва, 1979.

2. Ireland K., Rosen M., A classical introduction to modern number theory. Springer, New York, 1982.

Статья поступила 15.06.2005. 